\title{
La ética: más que un elemento integrador del proceso editorial
}

\author{
Ethics: More than an Integrating Element of the Editorial Process \\ A ética: mais do que um elemento integrador do processo editorial
}

DOI: 10.5294/aqui.2015.15.3.1

A la luz de la filosofía, la palabra "cuidado" viene del latín cogitare, que significa pensar. En la Edad Media, cuidar significaba siempre pensar, reflexionar, juzgar. Este énfasis reflexivo se ha ido perdiendo y se ha acentuado un sentido asistencial, protector y de ayuda. Este es el sentido del cuidado para Enfermería (1).

Cuidar es una actividad de reflexión y crítica que implica conocer y discernir... algo propio de la disciplina.

La palabra cuidado nos evoca significados, actitudes, acciones y relaciones, todo ello alrededor de la ética como elemento integrador.

Los términos ética y moral comparten, en cierto modo, sus etimologías. En la cotidianidad se utilizan para expresar igual sentido. La palabra "ética" viene del griego ethos, que significa costumbre o hábito, y "moral” viene del latín, que tiene el mismo significado.

La ética se considera una rama de la filosofía que estudia los comportamientos en cuanto pueden ser correctos o incorrectos, se relaciona con la moral y el actuar del ser humano.

¿Por qué esta introducción para abordar la ética como elemento integrador del proceso editorial de revistas científicas?

Porque están íntimamente relacionados; publicamos para el desarrollo de la disciplina de enfermería. La divulgación y visibili- dad de los trabajos de investigación de docentes e investigadores no solo aportan al desarrollo teórico, sino que contribuyen al mejoramiento de la calidad de vida de hombres y mujeres.

La ética está presente en toda la producción de una revista científica y compromete a cada uno de los actores que intervienen en ella. No es exclusiva de autores, también compromete al editor, a evaluadores y árbitros, a comités editoriales y científicos; igualmente, a quienes adelantan los procesos editoriales.

Con el fortalecimiento y la expansión de redes de investigación, gracias a las tecnologías de información y comunicación (TIC), que rompen y superan fronteras geográficas, culturales y de lenguaje, la ética se convierte en la médula de cualquier diálogo y dinámica integradora entre académicos e investigadores.

En América Latina, como en los centros de investigación más avanzados del mundo, hemos pasado en pocas décadas a acciones de cooperación, alianzas estratégicas y convenios con enfoques multidisciplinarios. Son redes complejas y dinámicas. Las universidades se han acercado al sector real, con el modelo universidad-empresa, y participación activa de la sociedad (2).

Los escenarios de investigación se salen del aislamiento de los laboratorios, como lugares privados y herméticos. El laboratorio está ahí, en la misma sociedad. Es en ese lugar en donde ocurren los fenómenos de estudio y, de manera particular, los fenómenos alrededor del cuidado de enfermería, del cuidado de la salud. 
Y es justo en ese escenario, en donde la ética de los investigadores enfrenta sus primeros retos, al realizar trabajos de campo, y estudios de caso y cualitativos. Se trata del consentimiento informado, entendido como la manifestación expresa de una persona de participar de manera libre y voluntaria en el estudio. Este es resultado del cambio cultural y la evolución de la relación entre el investigador y las personas o los grupos participantes, reconociendo la autonomía y la responsabilidad de la práctica investigativa.

De manera que la práctica y los procesos de investigación no están exentos de conflictos éticos; conflictos que, a su manera y circunstancias, debe afrontar igualmente el editor.

Son muchos los dilemas éticos de un editor; entre ellos, podríamos mencionar la validez del proceso investigativo y sus resultados a través del tiempo, las dificultades o la capacidad para comprender los resultados de determinados estudios y, en ocasiones, la desconfianza por aspectos no siempre transparentes.

Alrededor de una revista científica, se tejen una serie de procesos complejos sobre los cuales la comunidad académica espera altos niveles de calidad, transparencia, rigor y credibilidad, aspectos que no se logran si se incurre en faltas éticas (3).

Entre los protagonistas de primer orden en la edición científica están los evaluadores, a quienes se les ha dado el título de "certificadores del conocimiento". Ellos, los aseguradores de la calidad de los contenidos de una revista, son seleccionados bajo altas condiciones personales y académicas, y representan lo más autorizado de las comunidades científicas de una disciplina, por su trayectoria, reconocimiento y prestigio. Entonces, se espera de ellos un exigente trabajo, cuyo resultado, también, es sometido al escrutinio de la comunidad científica.

El evaluador debe analizar el trabajo con los criterios de originalidad, pertinencia y validez, al igual que con el mayor rigor para poder identificar los posibles conflictos de interés que, si no son resueltos, derivan en graves faltas éticas.

Pero la tarea y responsabilidad del evaluador van más allá del concepto de aceptado o rechazado; sus conceptos y sugerencias, también, contribuyen a la reflexión y argumentación temática (4).

Ahora bien, ¿qué decir acerca de la relación entre resultados de investigación y práctica de enfermería?
¿Está la práctica de enfermería guiada por la ciencia?

¿Se incorporan de manera frecuente los resultados de los estudios?

¿Conocen los enfermeros(as) de servicio en las áreas clínicas y asistenciales estos resultados?

¿Qué tanto contribuyen los resultados publicados en las revistas científicas, en la realidad de los servicios asistenciales?

El esfuerzo de las publicaciones seriadas se ve altamente compensado, en la medida en que los resultados de novedosos y pertinentes estudios lleguen a la práctica para fortalecerla y transformarla. Sin embargo, existe una brecha muy grande entre el momento en que se producen los resultados de los proyectos de investigación y la incorporación de estos en la práctica.

Desde el punto de vista ético, debemos hacer estas preguntas e intentar responderlas. La responsabilidad es conjunta, también de quienes estamos en el medio académico, en la formación de nuevos profesionales de programas de pre y posgrado.

El principal reto del investigador en enfermería es lograr que los resultados de sus estudios y trabajos impacten y transformen la práctica.

Una buena estrategia para estimular e incrementar la consulta y aplicación de estos resultados por parte de enfermeros y enfermeras de áreas clínicas y asistenciales es ampliar las redes de trabajo y cooperación, incorporando a estos profesionales en grupos y proyectos de investigación, conjuntamente con sus resultados.

No debemos olvidar que los fenómenos de estudio del cuidado están en la práctica. Es allí donde suceden las principales acciones, por tanto, debemos incentivar que desde estos espacios se identifiquen los temas y problemas de investigación.

Escenarios clínicos, asistenciales, comunitarios, escolares y de salud ocupacional, por mencionar algunos, son espacios reales y cotidianos donde encontramos los problemas por resolver, con acciones de cuidado y educación.

María Clara Quintero Laverde Decana, Facultad de Enfermería y Rehabilitación 


\section{Referencias}

1. Botero UD. Si la Naturaleza es sabia, el hombre no lo es. El Cuidado de la vida a la luz de la Filosofía. Cátedra Manuel Ancizar Cuidado de la Vida. Bogotá: Universidad Nacional; 2006. p. 15-34.

2. Organización para la Cooperación y el Desarrollo Económico (ODCE)-Banco Mundial (BM). Investigación e Innovación. En: Evaluación de Políticas Nacionales de Educación. La Educación Superior en Colombia. Bogotá: OCDE-BM; 2012.

3. Bravo TR. Aspectos éticos en las publicaciones científicas. [Citado 2015 agosto 18]. Disponible en: http://www.infodoctor. org/rafabravo/fraude.htm

4. Ramírez DC, Martínez LC et al. Divulgación y difusión del conocimiento: las revistas científicas. Bogotá: Universidad Nacional de Colombia; 2012. 